\title{
Estimating SARS-CoV-2 infection fatality rate using data from one of the most affected areas worldwide
}

\author{
Giovanni Landoni ${ }^{1,2, *}$, Carolina Faustini ${ }^{1}$, Marilena Marmiere ${ }^{1}$, \\ Carolina Soledad Romero Garcìa ${ }^{3,4}$, Annalisa Ruggeri ${ }^{5}$, Alberto Zangrillo ${ }^{1,2}$
}

\author{
${ }^{1}$ Department of Anesthesia and \\ Intensive Care, IRCCS San Raffaele \\ Scientific Institute, 20132 Milan, Italy \\ ${ }^{2}$ Faculty of Medicine, Vita-Salute San \\ Raffaele University, 20132 Milan, Italy \\ ${ }^{3}$ Hospital General Universitario de \\ Valencia, 46014 Valencia, Spain \\ ${ }^{4}$ Division of Research Methodology, \\ European University, Valencia, Spain \\ ${ }^{5}$ Hematology and Bone Marrow \\ Transplant Unit, IRCCS San Raffaele \\ Scientific Institute, 20132 Milan, Italy \\ *Correspondence \\ landoni.giovanni@hsr.it \\ (Giovanni Landoni)
}

\begin{abstract}
The real estimate of the infection fatality rate of SARS-CoV-2 is a pivotal aspect of the COVID-19 pandemic. However, this number is still debated, since both the numerator and the denominator are uncertain. Data analysis from the most affected areas in the world minimizes computational errors and represents a unique approach for estimating infection fatality rate. We first extracted data from PubMed/Medline, Google, traditional media and social media to obtain the rate of SARS-CoV-2 antibodies seroprevalence in the most affected and best-studied areas in the world: Val Seriana (Italy), Ischgl (Austria) and Manaus (Brasil). We then searched mortality data from national institutes of statistics and calculated excess mortality. We estimated the infection fatality rate considering several scenarios according to the mortality attributable to COVID-19 and the proportion of the population infected with the virus. We found that the seropositivity was surprisingly close to $40 \%$ in all the considered areas. We calculated the SARS-CoV2 infection fatality rate for Val Seriana, using from half to the entire excess mortality (1208 deaths) and considering from $40 \%$ to $80 \%$ of the population as being infected with SARS-CoV-2. In the most conservative scenario, infection fatality rate was as low as $0.55 \%$, while in the worst-case one it was $2.2 \%$. We found that the seroconversion rate in the most affected areas worldwide is about $40 \%$. We consequently estimated the infection fatality rate to be between $0.55 \%$ and $2.2 \%$ in an area with a relatively elderly population.
\end{abstract}

\section{Keywords}

SARS-CoV-2; Infection fatality rate; Antibodies; COVID-19; Mortality; Lethality

\section{Introduction}

Coronavirus disease 2019 (COVID-19) is a global pandemic affecting 220 countries and more than 220 million patients worldwide, which caused more than 4.5 million deaths as of September 5th 2021 [1]. This disease is caused by SARS-CoV2 , firstly identified in a cluster of patients with extended and unexplained pneumonia in Wuhan, China.

The infection fatality rate of SARS-CoV-2 is still debated, with uncertainties in the number of patients who died and the number of patients who got infected with the virus. The infection fatality rate is the proportion of deaths from a specific disease compared to the total number of infected individuals over a certain period of time [2]. One of the main issues when calculating the case fatality rate is to identify the exact numerator and denominator. This is especially true for COVID19 , which is characterized by a variety of presentations, going from asymptomatic to disease requiring hospitalization and intensive care. Data analysis from the most affected areas represents an innovative method for estimating the infection fatality rate, since the number of individuals infected with the virus approaches the total population, minimizing errors in the denominator. Moreover, in these areas, the excess mortality is extensively studied and represents a good approximation of the number of COVID-19 deaths.

After the outbreak in China, Italy was the first country in Europe to be overwhelmed by COVID-19 and has more than 4.5 million laboratory-confirmed cases and 129,466 reported deaths to date [1]. Val Seriana, a highly industrialized valley in Lombardy with 136,194 residents, was the most affected and well-studied area in Italy, where the first identified case was registered on February 23rd 2020, in Alzano Lombardo [3].

In the same period, a ski resort in Ischgl, Austria, became the most famous COVID-19 epicenter in Europe [4]. A bartender was tested positive for SARS-CoV-2 on March 7th 2020, and that subsequently led to a significant percentage of the 1580 inhabitants being infected. The infection spread far and wide due to tourists carrying the virus back into their own countries, linking Ischgl to cases spread over 45 nations.

Brazil experienced one of the world's most rapid-growing COVID-19 epidemics. Manaus is the largest metropolis in the the Amazon region, with over two million people and a population density of 158 inhabitants $/ \mathrm{km}^{2}$ [5]. The first SARS-CoV-2 case in Manaus was confirmed on March 13th 
2020, and was followed by a violent epidemic.

In the hypothesis that these well-known and best-studied areas were also those with the highest proportion of SARSCoV-2 positivity, we looked for seroconversion and excess mortality data and tried to estimate the infection fatality rate.

\section{Methods}

We searched data from PubMed/Medline, Google, traditional media and social media to obtain seroprevalence of SARSCoV-2 antibodies in Val Seriana, Ischgl and Manaus. We chose these three areas because they experienced extraordinary and dramatic COVID-19 outbreaks and because the presence of antibodies against SARS-CoV-2 was documented with reliable immunoassays, such as chemiluminescent microparticle immunoassay (CMIA) and enzyme-linked immunosorbent assay (ELISA).

We extracted mortality data from the Italian National Institute of Statistics and compared them with the previous four years. We calculated excess mortality subtracting the average number of deaths between March 1st and May 1st 2015-2019 to the raw number of fatalities between March 1st and May 1st 2020.

For what concerns the numerator, we hypothesized the number of COVID-19 deaths to be between half and the whole excess mortality, since there is debate whether the excess mortality should all be attributed to COVID-19 or not [6].

We then used both the number of seropositive individuals and double the number of seropositive individuals as the denominator, representing the number of people infected with SARS-CoV-2, since a relevant part of individuals exposed to SARS-CoV-2 neutralizes the virus at the mucosal level and develops specific memory $\mathrm{T}$ cells with a highly active cytotoxic phenotype $[7,8]$.

The infection fatality rate was obtained by dividing the number of deceased individuals attributed to SARS-CoV-2 by the number of subjects who were supposed be infected with the virus.

Infection fatality rate $=\frac{\text { mortality attributed to COVID-19 }}{\text { subjects who encountered the virus }}$

We therefore calculated four possible scenarios, with the more conservative one having half the excess mortality as the numerator and double the number of seropositive as the denominator and the most worrying one having the whole excess mortality as the numerator and the number of seropositive as the denominator.

$$
\begin{gathered}
\text { Best-case scenario }=\frac{\text { half excess mortality }}{\text { double the number of seropositive }} \\
\text { Intermediate-case scenarios }=\frac{\text { half excess mortality }}{\text { number of seropositive }} \\
=\frac{\text { excess mortality }}{\text { double the number of seropositive }} \\
\text { Worst-case scenario }=\frac{\text { excess mortality }}{\text { number of seropositive }}
\end{gathered}
$$

Since the infection fatality rate is strongly associated with age, we also extracted the median age of the studied population from the National Institute of Statistics.

\section{Results}

Surprisingly, the rate of antibody positivity was similar in the three locations and close to $40 \%$. Immunoglobulins were detectable in $44 \%$ of the population in Manaus [5] and in $42.2 \%$ in Ischgl in April 2020 [9]. In Val Seriana, we found 38\% of 423 workers had antibodies for SARS-CoV-2 in May [10], while $42.3 \%$ of the 22,559 volunteers who underwent serological screening tested positive in July 2020 [11]. The methods used for antibody detection were chemiluminescent microparticle immunoassay (CMIA; AdviseDx, Abbott, sensitivity = 98.1\%, specificity $=99.6 \%$, positive predictive value $\geq 92.8[95 \%$ Confidence Interval (CI): 85.8\%; 96.3\%], negatitive predictive value $\geq 98.1$ [95\% CI: 90.7\%; 99.7\%]) in Manaus and enzymelinked immunosorbent assay (ELISA; DIA.PRO, sensitivity = $66.3 \%$, specificity $=97.1 \%$, positive predictive value $=97.1$ [95\% CI: 94.6\%; 99.6\%], negatitive predictive value $=66.7$ [95\% CI: 59.6\%; 73.7\%]) in Ischgl and Val Seriana.

We found detailed data on excess mortality for Val Seriana only, and we therefore restricted to that region the evaluation of the COVID-19 infection fatality rate. The 38 municipalities included in the analyses are shown in Supplementary Table 1, together with their excess mortality. The National Institute of Statistics [12] reported a 503\% mean increase in the number of deaths in the considered time interval compared to the previous four years $(n=1448$ vs $n=240)$ in Val Seriana, with the highest percentages observed in the more densely populated municipalities (1125\% in Nembro, and $1030 \%$ in Alzano Lombardo, in March 2020). According to these data, we calculated the infection fatality rate range of COVID-19 considering different scenarios having, as denominator, $40 \%$ and $80 \%$ of the population.

In the most conservative scenario, the infection fatality rate was as low as $0.55 \%$.

$$
\frac{\text { half excess mortality }}{80 \% \text { population }}=\frac{604}{108,955}=0.55 \%
$$

On the opposite, considering the worst-case scenario, the infection fatality rate was $2.2 \%$.

$$
\frac{\text { total excess mortality }}{40 \% \text { population }}=\frac{1208}{54,478}=2.2 \%
$$

The intermediate-case scenarios, which consider total vs half the excess mortality as the numerator and number of seropositive vs double the number of seropositive as the denominator, have a case-fatality rate of $1.1 \%$.

$$
\begin{aligned}
& \frac{\text { total excess mortality }}{80 \% \text { population }}=\frac{1208}{108,955}=1.1 \% \\
& \frac{\text { half excess mortality }}{40 \% \text { population }}=\frac{604}{54,478}=1.1 \%
\end{aligned}
$$

These estimates of the infection fatality rate were obtained 
in an area with a median age of the general population of 43 years. In Italy, the median age is 45 years and the life expectancy is 83 years. According to data from Istat, $1 \%$ of the Italian population has an age between $90-94,2.3 \%$ of the Italian population has an age between $85-89,3.6 \%$ of the population has an age between $80-84$ years, $4.5 \%$ of that has an age between $75-79$ and $5.4 \%$ of the population has an age between 70-74 [13].

\section{Discussion}

We found that the SARS-CoV-2 seroconversion rate was remarkably overlapping in the most affected areas of the world and in the range of 38-42.2\% between April 2020 and July 2020 [7-9]. With this high seroprevalence and the subsequent minimization of computational errors, we estimated the infection fatality rate to be in the range of $0.55-2.2 \%$ in Val Seriana, an area characterized by an elderly population.

The seroconversion rate overlap among the most affected areas in the world can be interpreted either as temporary herd immunity or as the threshold above which strict lockdown strategies became mandatory (or personal behavior changed dramatically). Considering the short period in which such rate of SARS-CoV-2 seropositivity was reached, it is likely that the transmissibility index $\mathrm{R}_{0}$ was higher than initially estimated by the World Health Organization. There was probably an underestimation of the degree of viral spreading in the reports suggesting a $R_{0}$ of 3 in Lombardy at the end of February 2020 [14] and $a R_{0}$ of 2.2 in Brazil at the end of March 2020 [15]. A $40 \%$ seroconversion rate indicates a considerable spread of the virus, which presumably reached every household in these areas.

We suggest an innovative approach to estimate the infection fatality rate, which applies to areas with a very high spread of the virus since it minimizes errors in both the numerator and the denominator. We used excess mortality as the numerator, a reliable estimate of the number of subjects who died of COVID-19. It is a more specific measure of the pandemic's impact compared to the confirmed COVID-19 death count alone. On the other hand, recognizing that not all the excess mortality can be attributed to COVID-19, we added a best-case scenario with only half the excess mortality at the numerator. Moreover, since we applied our analyses only to the most affected areas worldwide, the denominator increases and, even if the exact number of infected people (numerator) still isn't precise, errors in the calculation of infection fatality rate are minimized. A significant proportion of asymptomatic or mild-symptomatic subjects could have developed a specific cytotoxic $\mathrm{T}$ cell response to the virus: these patients did not develop antibodies and therefore might not be traced through screening procedures [8].

Other authors calculated the overall case fatality rate of confirmed COVID-19 cases in the Italian population to be $7.2 \%$ (1625 deaths/22,512 cases) in March 2020 [16]. The authors gave three possible explanations for this result: the Italian population is elderly; all subjects testing RT-PCR positive for SARS-CoV-2 who died were considered COVID-19 deaths; and the Italian Ministry of Health used stringent testing policy, reducing the denominator. This study has several differences compared to ours: it produced a single estimate, while we calculated a numerical range, according to different scenarios. Moreover, Onder et al. [16] used the number of confirmed SARS-CoV-2 subjects who died-regardless of the preexisting diseases that may have caused death - as the numerator. Excess mortality, on the other hand, is considered a more precise estimate and a more comprehensive measure of the impact of the pandemic on deaths, rather than the confirmed COVID-19 death count alone [6]. Interestingly, we found a relatively low infection fatality rate, considering that the calculation was based on an area characterized by an elderly population.

Our study has strengths and limitations. We initially used report from traditional media and social media, but the majority of the findings were later published in peer reviewed journals. The methods used (CMIA or ELISA) for diagnosis of SARS$\mathrm{CoV}-2$ infection might have had an impact on the percentage of individuals exhibiting SARS CoV-2 antibodies. The infection fatality rate was obtained for one single area since Val Seriana was the only one with detailed excess mortality data, and might have limited worldwide applicability especially for countries with less exhaustive epidemiology registries and with a younger population. Nonetheless, our methodology can be easily applied when data on excess mortality in Ischgl and Manaus will be available, or in other area also taking into consideration different test performance evaluations. In addition, since the prevailing SARS-CoV-2 variant during the first phase of the pandemic has been replaced by variants with higher infectivity, fatality rates might have changed. Future studies can also attempt to replicate our findings in different areas where the population was affected by the SARS-CoV-2 epidemic, and we anticipate that these results will be dependent on the median age of the population, but also from the different times of recording and adherence to mask mandates and social distance which could reduce the viral load.

\section{Conclusions}

Our study aimed at addressing one of the most significant issues of the SARS-CoV-2 pandemic: the infection fatality rate. Indeed, COVID-19 was able to bring down entire nations, even if our calculations suggested a relatively low infection fatality rate, ranging from 0.55 to $2.2 \%$, in a highly affected area characterized by an elderly population. We also proposed a method to replicate these calculations in other highly affected areas. We also found a surprisingly high and overlapping SARS-CoV-2 seroconversion rate, around $40 \%$, in the world's most affected regions where reliable assays were used.

\section{AUTHOR CONTRIBUTIONS}

GL, CF, MM, AZ designed the research study. GL, CF, MM, $\mathrm{CS}, \mathrm{AR}$ collected data. GL, CF, MM, AZ analyzed the data. GL, CF, MM, AR wrote the manuscript and provided critical review. GL and AZ provided administrative support. All authors read and approved the final manuscript. 


\section{ETHICS APPROVAL AND CONSENT TO PARTICIPATE}

Not applicable.

\section{ACKNOWLEDGMENT}

We thank Zaraca Luisa, MD and Piersanti Gioia, medical student for carefully revising the manuscript and Nakhnoukh Cristina Msc and Bonizzoni Matteo Aldo, MD for their continuous support.

\section{FUNDING}

This research received no external funding.

\section{CONFLICT OF INTEREST}

The authors declare no conflict of interest. Giovanni Landoni and Carolina Soledad Romero Garcìa are members of the Editor Board of the journal.

\section{SUPPLEMENTARY MATERIAL}

Supplementary material associated with this article can be found, in the online version, at https://oss.signavitae. com/mre-signavitae/article/1435420442250625024/ attachment/Supplemental\%20material.pdf.

\section{DATA AVAILABILITY}

The data used to support the findings of this study are available from the corresponding author upon request.

\section{REFERENCES}

[1] Johns Hopkins University Medicine. COVID-19 Dashboard by the Center for Systems Science and Engineering (CSSE) at Johns Hopkins University of medicine. Available at: https://coronavirus.jhu. edu/map.html (Accessed: 5 September 2021).

[2] World Health Organization. Estimating mortality from COVID-19 Scientific Brief. 2020. Available at: https://www.who.int/newsroom/commentaries/detail/estimating-mortality-fromcovid-19 (Accessed: 5 September 2021).

[3] BergamoNews. Bassa Val Seriana, sierologici a tappeto: su 22mila abitanti positivo il 42.3\%. 2020. Available at: https: //www. bergamonews.it/2020/10/02/bassa-val-serianasierologici-a-tappeto-su-22mila-abitanti-positivo-il423/396601/ (Accessed: 1 August 2021).

[4] Coronavirus: 'Momentous' errors worsened Austria ski resort outbreak. 2020. Available at: https://www.bbc.com/news/world-europe54523014 (Accessed: 5 September 2021).
[5] Buss LF, Prete CA, Abrahim CMM, Mendrone A, Salomon T, de Almeida-Neto C, et al. Three-quarters attack rate of SARS-CoV-2 in the Brazilian Amazon during a largely unmitigated epidemic. Science. 2020; 371: 288-292.

[6] Our World in Data. Excess mortality during the Coronavirus pandemic (COVID-19). 2021. Available at: https://ourworldindata.org/ excess-mortality-covid (Accessed: 5 September 2021).

[7] Channappanavar R, Fett C, Zhao J, Meyerholz DK, Perlman S. Virusspecific memory CD8 $\mathrm{T}$ cells provide substantial protection from lethal severe acute respiratory syndrome coronavirus infection. Journal of Virology. 2014; 88: 11034-11044.

[8] Sekine T, Perez-Potti A, Rivera-Ballesteros O, Strålin K, Gorin JB, Olsson A, et al. Robust T Cell Immunity in Convalescent Individuals with Asymptomatic or Mild COVID-19. Cell. 2020; 183: 158-168.e14.

[9] Knabl L, Mitra T, Kimpel J, Rössler A, Volland A, Walser A, et al. High SARS-CoV-2 seroprevalence in children and adults in the Austrian ski resort of Ischgl. Communications Medicine. 2021; 1: 4.

[10] Perico L, Tomasoni S, Peracchi T, Perna A, Pezzotta A, Remuzzi G, et al. COVID-19 and lombardy: TESTing the impact of the first wave of the pandemic. EBioMedicine. 2020; 61: 103069.

[11] L'ECO DI BERGAMO. Val Seriana, il 42.3\% a contatto col virus - Più colpiti dai 45 anni in su e le donne. 2020. Available at: https://www. ecodibergamo.it/stories/bergamo-citta/valseriana-il-423-a-contatto-col-viruscovid-i-risultatidei-test-sierologic_1372521_11/(Accessed: 1 August 2021).

[12] ISTAT - Istituto Nazionale di Statistica. L'andamento dei decessi nel periodo 1 gennaio - 31 dicembre per gli anni 2015-2020. Dati anticipatori per l'anno 2020 sulla Base dati integrata della mortalità giornaliera comunale. Available at: https://public.tableau.com/views/ Variazioni/DecessiIstat?: language=it\&:display_count=y\& publish=yes\&: origin=viz_share_link\&: showVizHome=no\#3 (Accessed: 1 August 2021).

[13] tuttitalia.it. Popolazione per età, sesso e stato civile 2019. 2019. Available at: https://www.tuttitalia.it/statistiche/popolazioneeta-sesso-stato-civile-2019 (Accessed: 1 August 2021).

[14] Ministero della Salute. Covid-19, studio ISS: ecco quando un malato contagiava tre persone. 2020. Available at: http: //www.salute.gov.it/portale/news/p3_2_1_1_1.jsp? lingua=italiano\&menu=notizie\&p=dalministero\&id=4460 (Accessed: 5 September 2021).

[15] Sabino EC, Buss LF, Carvalho MPS, Prete CA, Crispim MAE, Fraiji NA, et al. Resurgence of COVID-19 in Manaus, Brazil, despite high seroprevalence. The Lancet. 2021; 397: 452-455.

[16] Onder G, Rezza G, Brusaferro S. Case-Fatality Rate and Characteristics of Patients Dying in Relation to COVID-19 in Italy. Journal of the American Medical Association. 2020; 323: 1775-1776.

How to cite this article: Landoni $G$, Faustini $C$, Marmiere M, Romero Garcìa CS, Ruggeri A, Zangrillo A. Estimating SARS-CoV-2 infection fatality rate using data from one of the most affected areas worldwide. Signa Vitae. 2021;17(5):30-33. doi:10.22514/sv.2021.149. 